\title{
Special issue: selected papers from NORCHIP 2014 conference
}

\author{
Timo Rahkonen ${ }^{1}$
}

Published online: 24 July 2015

(c) Springer Science+Business Media New York 2015

32nd Norchip Conference was held at Tampere, Finland in October 2014. The Nordic Microelectronics event has been the annual meeting-place of Nordic IC and ASIC designers since 1983, when it was first held in Copenhagen. Already in early 1980s MPW runs were constantly arranged for Nordic research institutes and this helped to educate the designers needed for the 1990s telecommunication boom, for example. Since 1992 Springer (initially Kluwer) has published a special issue containing the best papers of the conference.

Now Norchip conference is following the path of ESSCIRC: due to gradually reducing number of attendants, it will change to a combined conference with a bit broader scope. In 2015 the meeting will not be called Norchip anymore, but joined together with SoC conference, it will continue under a name of NORCAS (IEEE Nordic Circuits and Systems Conference). So NORCHIP is signing out, but warmly welcomes NORCAS to continue as a meeting forum of IC and ASIC designer. Interested readers are welcomed to read a short history of the conference at http:// www.norchip.org/NORCHIP_HISTORY.htm and submit their papers to NORCAS (http://www.norcas.org/).

The strength of Norchip has always been that it has plenty of presentations showing fabricated and tested circuits. For this special issue I chose eight interesting papers that I'll introduce now briefly.

Timo Rahkonen

timor@ee.oulu.fi

1 Electronics Laboratory, University of Oulu, 90014 Oulu, Finland
"Integrated Reconfigurable High-Voltage Transmitting Circuit for CMUTs" by Muntal et al. presents a highvoltage driver circuit for capacitive micromachined ultrasonic transducers (CMUT). Implementing a large array of transducers for a portable ultrasound scanner forced the designers to minimize the power needed in the level translating circuit and carefully choosing proper type of transistors for the partially high-voltage application.

"A $4 \times 128$ SPAD array with a 78-ps 512-Channel TDC for time-gated pulsed Raman spectroscopy" by Nissinen et al. is another high-voltage application, where pn diodes are biased in avalanche breakdown region to implement very high-gain photo-receivers, where a single photon is sufficient for triggering a timing pulse in a complete long-range, low-power laser radar.

"Integrated 16-Channel Neural Recording and Stimulation Circuit" by Kursu et al. shows the implementation of a system for real-time recording of neural signals that may eventually help us to understand what cockroaches really think about human beings or life in general.

"A novel cascading scheme to improve the performance of voltage multiplier circuits" by Chouchan et al. is a timely implementation of an RF energy harvester circuit operating at $433 \mathrm{MHz}$. The active rectifying circuit was modified to give higher efficiency at high input powers.

“A $28 \mathrm{GHz}$ SiGe PLL for an 81-86 GHz E-band beam steering transmitter and an I/Q phase imbalance detection and compensation circuit" by Tired et al. describes a twochip PLL system and preliminary results for controlling the phase of a $80 \mathrm{GHz}$ transmission. It is designed using $1.5 \mathrm{~V}$, $200 \mathrm{GHz}$ SiGe HBT process.

"W-Band Phase Shifter in 28-nm CMOS" by Vahdati et al. is another paper related to electrical beam-steering applications. It presents a 4-step controllable, $39 \mathrm{~mW}, 1-\mathrm{V}$ phase shifter for $80-100 \mathrm{GHz}$ band. 
"A low-power 2nd-order CT delta-sigma modulator with an asynchronous SAR quantizer" by Radjen et al. presents an interesting low-power AD converter architecture where a 2nd order loop filter is implemented by just one opamp, and multibit SAR quantization is done asynchronously to minimize the needed clock frequency.

Finally, another way of speeding up SAR conversion is shown in "A 10 bit 16-to-26 MS/s Flexible Window SAR ADC for Digitally Controlled DC-DC Converters in $28 \mathrm{~nm}$ CMOS" by Haenzche et al. Here, the successive approximation search can be initiated near the expected value, resulting in shorter conversion time in tightly fed-back digital controllers of DC-DC converters.

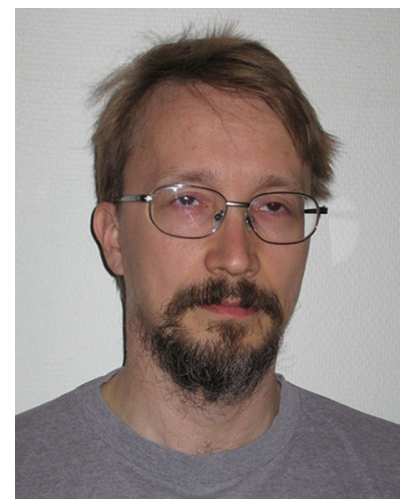

Timo Rahkonen is a professor in circuit theory and circuit design at the University of Oulu, Oulu, Finland. He has done research on CMOS delay line circuits, distortion memory effects in RF power amplifiers, and distortion contribution analysis. In general he is interested in linearizing the nonlinear effects in analog circuits. 